\author{
The Grateful Don't Cheat: \\ Gratitude as a Fount of Virtue \\ David DeSteno, Fred Duong, Daniel Lim, Shanyu Kates \\ Northeastern University
}

Corresponding Author:

David DeSteno

Department of Psychology

Northeastern University

Boston, MA 02115

Email: d.desteno@gmail.com

Date: January 31, 2019

In press, Psychological Science 


\begin{abstract}
Gratitude has been linked to behaviors involving the exchange of resources. As such, it motivates people to repay debts to benefactors. However, given its links to self-control - itself a necessary factor for repaying debts - the possibility arises that gratitude might enhance other virtues unrelated to exchange that depend on an ability to resist temptation. Here, we provide an examination of gratitude's ability to function as a "parent" virtue by focusing on its ability to reduce cheating. Using real-time behavior-based measures of cheating, we demonstrate that gratitude, as opposed to neutrality and the more general positive emotional state of happiness, reduces cheating both in a controlled laboratory setting $(\mathrm{N}=156)$ and a more anonymous online setting $(\mathrm{N}=141)$. This finding suggests that not all moral qualities need not be studied in silos, but rather that hierarchies exist wherein certain virtues might give rise to seemingly unrelated others.
\end{abstract}

Keywords: gratitude, cheating, moral virtue, emotion 
For the Roman statesman and philosopher Cicero, gratitude held a unique position among the noble qualities a person could possess: it was not only the greatest of the virtues, but also the parent of the others (Cicero $54 \mathrm{BCE} / 2018$ ). While the notion that all virtues derive from gratitude is assuredly an overstatement, the possibility that this emotion might give rise to certain, seemingly unrelated virtues stands as an intriguing, and in many ways theoretically profound, possibility. Traditionally speaking, virtues have been studied in silos, with the potential of one to affect the emergence of others rarely being considered (for examples, see Peterson \& Seligman, 2004; Sinnott-Armstrong \& Miller, 2017). As a consequence, empirical verification that certain moral virtues can be founts from which others flow would not only open new avenues for theory refinement, but also offer new routes by which character development could be pursued.

Cicero's musings aside, emerging evidence supports the idea that gratitude might function in just this way. While gratitude's links to relationship formation and psychological wellbeing are well established (Algoe, 2012; Emmons, 2009), within the realm of social decision-making, evidence for its influence has been primarily limited to behaviors involving exchange (DeSteno, Condon, \& Dickens, 2016). For example, gratitude has been shown to increase efforts to pay benefactors back for previous assistance (Bartlett \& DeSteno, 2006; Tsang, 2006) and to nudge people to exchange resources equally with others making similar decisions (DeSteno, Bartlett, Baumann, Williams, \& Dickens, 2010).

Such outcomes for gratitude make good sense given its intrinsic ties to elicitors involving exchange. Recent work, however, has also linked gratitude to self-control. For example, people feeling grateful, compared to others feeling happy or no emotion at all, show enhanced self- 
control when it comes to making financial decisions requiring patience to obtain larger rewards (DeSteno, Li, Dickens, \& Lerner, 2014; Dickens \& DeSteno, 2016). While it is certainly true that self-control is necessary for successful exchange and cooperation (Frank, 1988), the ability to delay gratification also plays a role in managing many other types of moral challenges. For this reason, we believe gratitude might function to enhance certain moral actions outside the realm of exchange - actions that are also characterized by the need to resist a temptation to satisfy a selfish urge for immediate gratification.

To examine this possibility, we focused here on the temptation to cheat, both in a context where doing so offers a way to avoid an onerous task (Study 1) and a way to obtain more money than one might truly deserve in a game of chance (Study 2). These situations present moral dilemmas that pit desires for pleasure or gain against universally accepted norms for honest behavior. Our straightforward prediction was that gratitude should reduce cheating in both cases.

\section{EXPERIMENT ONE}

In this experiment, we adapted a methodology we have previously used to study cheating (Valdesolo \& DeSteno, 2007, 2008) to examine how gratitude might affect people's decisions to behave ethically. As described in detail below, after inducing one of three emotional states in participants, we presented them with two different tasks, only one of which they needed to complete. The first was relatively short and fun; the second was much longer and onerous. We also gave them a device which purportedly would randomly determine which task they should do - in essence, a virtual coin flip. Unbeknownst to participants, however, the device was rigged such that it would always initially indicate that they were assigned to complete the longer and more difficult task. We then left them alone to complete their work. 
As part of this experiment, all participants therefore faced a moral conundrum. Should they in fact complete the more onerous task to which they had been assigned, or, given that they believed they had anonymity, pretend that they had been assigned to the easier, more enjoyable task? Our straightforward prediction was that participants experiencing gratitude would be less likely to cheat.

\section{Methods}

\section{Open Practices Statement}

The experiment presented here was preregistered as Study 1 in a multi-study project; preregistration files can be accessed at https://osf.io/qs6rk/. Data collection for the remaining studies is ongoing at the time of submission of this manuscript. Because the methods employed primarily involve real-time interactions with confederates and behavioral responses, the relevant materials and procedures are described in detail within the text of the manuscript. A link to a general instructional video for experiment's setup and the gratitude induction can be found at https://youtu.be/1O7XdPmBnfs. Detailed scripts can be obtained from the first author upon request.

\section{Participants}

As noted in the preregistration for this experiment, we initially intended to recruit 120 participants. This target was based upon two factors. The first was the desire to achieve a power $=.80$ based on an estimated effect size of Cohen's $d=.62$, which itself stemmed from an earlier published study examining the differential effects of gratitude and happiness on self-control (DeSteno et al., 2014). While only 105 participants would be needed to achieve this level of power, the experiment described here also served as a recruitment device for part of a larger longitudinal study (Study 4 in the aforementioned preregistration). Because we anticipated that 
not all participants would agree to take part in the subsequent study, and that some level of attrition would occur among those who did, we increased the number of participants to be recruited for this experiment to 120 (see preregistration).

As we conducted the experiment reported here, however, it became clear that the number of participants who either declined to take part in the longitudinal study or dropped out was greater than anticipated. As a result, we increased the number of participants in the current experiment until we reached the desired number for participation in the longitudinal study. Participants' decisions to take part in the longitudinal study were made after they completed the experiment reported here and thus could in no way influence the presents results. In addition, no data from this experiment (Study 1 in the pre-registration) were examined until all recruitment formally ended with a sample of 173 participants who were randomly assigned to one of three emotion induction conditions: gratitude, happiness, neutral. The result, as discussed below, provided a substantial increase in prospective power for this experiment.

Of the 173 participants, data from $17(9.8 \%)$ were removed from analysis because of issues of fluency in English, manipulation failure, or suspicion. As described below, the employed procedure relies upon deception (i.e., computers are perceived to malfunction) and the use of confederates who assist or otherwise interact with participants. If attempts at deception failed (e.g., the computer did not "crash" when intended or a participant believed she remedied the situation herself) or a participant had any pre-existing familiarity with a confederate or noted suspicion of any type during debriefing, his or her data were removed. The final sample consisted of 156 participants (gender: 113 women, 42 men, 1 unreported; Mean Age = 19 years, $\mathrm{SD}=1.06)$ 
Prospective power for this sample size using a Cohen's $w=.30$ (which is equivalent to a Cohen's $d=.62$, but more appropriate for contingency table analysis) is estimated to equal .93 . Cohen identified a $w=.30$ as a rule of thumb marker for a medium effect size for categorical data analysis. In addition to the rationale for this effect size given in the preregistration (i.e., based on a previous experiment from our lab), there is an additional reason why we believe it to be an appropriate effect size to use for prospective power calculations in the present case. Across five published experiments from our lab using different induction techniques and behavioral outcomes, the average effect size linking gratitude to prosocial behaviors falls between a medium and large effect size (see Bartlett \& DeSteno, 2006; DeSteno et al., 2010; DeSteno et al., 2014; Dickens \& DeSteno, 2016). Consequently, using the default Cohen's $w$ effect size for a medium effect seems prudent.

\section{Procedure}

The employed measures and procedures were reviewed and approved by the Northeastern University Institutional Review Board and conform to the provisions of the World Medical Association Declaration of Helsinki.

Participants believed they were one of two people taking part in the experiment. In actuality, the other individual was a confederate. When participants arrived, they were informed by the experimenter that the session would consist of three main parts: a test of general knowledge, which they would complete together, a test of word recognition which they would complete alone, and either a task involving a fun game or logic and math abilities, which they would also complete alone.

For the general knowledge task, participants worked with the confederate to answer a set of trivia-type questions. The sole purpose of this task was to provide a pretext for the subsequent 
measures of emotion in which people would be asked to report what they were feeling toward a partner. After the three minutes allotted for this task had passed, the experimenter collected the duo's responses which they believed would produce a joint score. In reality, scores were not calculated as they were irrelevant to the experiment. Next, participants moved to separate cubicles containing desktop computers for the word recognition task. Here, working individually, they were instructed to decide whether strings of letters flashed on the screen constituted words in English. They were told to make decisions as quickly and accurately as possible and that their scores would appear on the screen after the end of the third block of trials. This task was intended to be very tedious and time-consuming, as it required vigilance for many seemingly repetitive trials. The experimenter explained that when the task was over, he would manually record the score for each block. Although this task was completed in each of the emotion conditions, its purpose was solely to provide an aversive experience that would play a central role in the induction of gratitude.

At this point, the common procedural script diverged in the three emotion conditions. After completing the inductions, participants completed a manipulation check and were then confronted with the opportunity to cheat contained in what they believed was the third and final task of the session.

\section{Manipulations and Measures}

Gratitude Induction. The gratitude induction was adapted from a previously established paradigm (Bartlett \& DeSteno, 2006; DeSteno et al., 2010; Dickens \& DeSteno, 2016). It unfolded in the following manner. As participants waited for the computer on which they had just completed the word recognition task to calculate their final scores, it appeared to crash. In 
actuality, this crash was pre-programmed. For participants, however, the apparent result was that the results of their tedious work had disappeared.

The confederate, who ostensibly had completed her own tasks, looked over at the true participant to see what was happening. At this point, the experimenter entered the room, noticed the computer problems, and subsequently explained that a technician would be called to fix the computer. The experimenter also informed the participant that he or she would need to start the onerous task again from the beginning. While the experimenter went to call the technician, the confederate attempted to see if she could help the participant. Following a scripted series of comments and behaviors, the confederate tried to figure out what was wrong with the computer by hitting keys, playing with wires, and checking plugs. At a preset time, she surreptitiously hit a key that, after a brief interval, caused the computer screen to come on with the participant's scores being displayed, giving the impression that the computer was fixed by the confederate. The experimenter then allowed the participant to continue with the experiment from that point as opposed to completing the word recognition task again.

Happiness Manipulation. In this condition, participants did not experience a computer crash; their scores correctly displayed after completion of the word recognition task. The experimenter next directed respective pairs of participants and confederates to watch a twominute comedic video together that of a series of funny clips involving babies and animals. After watching the video, the confederates engaged participants in a brief discussion of their reactions to the clips. This interaction allowed for a verbal exchange similar in length as to what occurred in the gratitude condition (see Bartlett \& DeSteno, 2006).

Neutral Manipulation. In this condition, events mirrored those of the happy condition with the exception that the two-minute video consisted of clips from a documentary on geology. 
Emotion measures. As part of a questionnaire purported to assess reactions to the general knowledge task, participants answered questions regarding their emotional states and feelings toward their partner (i.e., the confederate). Responses were collected using a 5-point scale. Gratitude was assessed as the mean response to two items: "How grateful/appreciative do you feel toward your test of general knowledge partner?" Happiness was assessed as the mean response to two items: "How happy/amused are you feeling?"

Cheating. To measure participants' willingness to cheat, we adapted a paradigm utilized by Valdesolo and DeSteno $(2007,2008)$ in which participants can cheat on an assignment procedure in order to avoid having to complete an onerous task. Participants were first informed that they would need to complete one of two possible tasks: a 10-minute one involving a fun numbers game or a 45-minute one involving difficult math and logic problems. They were next informed that they were to use a randomizer at a separate computer to determine which task they were to complete. When they hit a key, it would flip a colored virtual coin. If it came up green, they would complete the short task; if it came up red, they would complete the long task. The experimenter told them that this procedure was being used to ensure equal and fair assignment to conditions. Every other participant would use this randomizer. He or she would go on to complete the indicated task, leaving the opposing task to be completed by the next participant. It was also made very clear to participants that this next participant was a stranger and not the confederate.

The experimenter then emphasized the confidential nature of the assignment procedure, noting that the randomizer was on a separate computer that did not record results and that the experimenter would leave the room before the participant flipped the coin. In addition, the computer instructions stated that the following participant would not know that the current 
participant had used the randomizer to assign conditions. The experimenter then demonstrated how the randomizer worked, showing that the coin could land on either green or red, and then left the room, leaving the participant to complete the assignment procedure and the indicated task.

Unbeknownst to participants, the randomizer was preset to always land on the 45-minute task the first 2 times it was used in a session. Participants were told that after using the randomizer, they were to hit the key for their assigned task and follow along with the instructions. Therefore, anyone who completed the short task cheated, either by intentionally disregarding the information from the randomizer or by refusing to use it at all. Of import, decisions to act in this way have been almost unanimously identified as a moral transgression. A survey of 42 individuals by Valdesolo and DeSteno (2008) found that fully $100 \%$ of people viewed a refusal to abide by the randomizer's flip as morally wrong. Likewise, a survey of 80 people by Batson and colleagues found $98 \%$ of individuals held a similar view (Batson, Kobrynowicz, \& Dinnerstein, 1997). After participants completed this third task, the experiment ended, followed by debriefing and probing for suspicion.

\section{Results}

\section{Manipulation Check}

We submitted participants responses on the gratitude and happiness intensity scales to a 3 (Emotion Condition: Gratitude, Happiness, Neutral) X 2 (Emotion Intensity: Gratitude vs. Happiness) mixed ANOVA. As expected, the emotion manipulations produced distinct states as indicated by a significant interaction, $F(2,153)=5.20, p=.007$. A one-way ANOVA on gratitude intensity proved significant, $F(2,153)=6.30, p=.002$, with Fisher's LSD comparisons $(\alpha=.05)$ confirming that those who completed the gratitude manipulation experienced 
significantly greater intensities of gratitude $(M=5.68,95 \%$ CI: $5.39-5.96)$ than did those who completed the happiness $(M=5.08,95 \% \mathrm{CI}: 4.79-5.37)$ and neutral manipulations $(M=4.94$, $95 \%$ CI: $4.57-5.31)$.

Turning to happiness intensity, a similar one-way analysis confirmed that those who completed the happiness $(M=4.74,95 \% \mathrm{CI}: 4.46-5.01)$ and gratitude $(M=4.65,95 \% \mathrm{CI}: 4.35$ - 4.95) manipulations reported greater intensities of happiness than did those who completed the neutral manipulation $(M=3.80,95 \% \mathrm{CI}: 3.42-4.18), F(2,153)=9.87, p<.001$. The fact that those in the gratitude and happiness conditions reported similar levels of happiness but distinct levels of gratitude is to be expected, as the experience of gratitude itself usually co-occurs with positive feelings regarding a benefit received (e.g., a gift, being relieved of a problem). Enhanced feelings of gratefulness in the presence of elevated levels of happiness has traditionally been used to indicate a discrete state of gratitude - one that, of import, demonstrates predictive validity by leading to behavioral outcomes that are distinct from than those stemming from happiness alone (Bartlett \& DeSteno, 2006; DeSteno et al., 2014).

\section{Cheating}

Frequencies for cheating and behaving fairly as a function of emotional state are shown in Table 1. A Fisher-Freeman-Halton exact test confirmed that an association between emotion and cheating was highly probable, with deviations in the proportion of cheating across emotion conditions as large or larger than that observed here only occurring by chance about one percent of the time $(p=.012)$. Examination of the adjusted standardized residuals clearly indicates that the principal deviations occurred among those feeling grateful, with the corresponding Pearson residuals pointing to the low number of cheaters in the gratitude condition as the primary discrepancy. 


\begin{tabular}{lcccccccc}
\cline { 2 - 7 } Emotion & $\begin{array}{l}\text { Observed } \\
\text { Frequency }\end{array}$ & $\begin{array}{l}\text { Expected } \\
\text { Frequency }\end{array}$ & $\begin{array}{l}\text { Pearson } \\
\text { Residual }\end{array}$ & $\begin{array}{l}\text { Adj. Std. } \\
\text { Residual }\end{array}$ & $\begin{array}{l}\text { Observed } \\
\text { Frequency }\end{array}$ & $\begin{array}{l}\text { Expected } \\
\text { Frequency }\end{array}$ & $\begin{array}{l}\text { Pearson } \\
\text { Residual }\end{array}$ & $\begin{array}{l}\text { Adj. Std. } \\
\text { Residual }\end{array}$ \\
\hline \multirow{2}{*}{ Gratitude } & 1 & 6.2 & -2.1 & -2.8 & 53 & 47.8 & 0.8 & 2.8 \\
Neutral & 7 & 5.0 & 0.9 & 1.1 & 36 & 38.0 & -0.3 & -1.1 \\
Happiness & 10 & 6.8 & 1.2 & 1.6 & 49 & 52.2 & -0.4 & -1.6 \\
\hline
\end{tabular}

Table 1. Cheating as a Function of Emotion Condition in Experiment 1.

In order to determine whether infrequent cheating in the gratitude condition explained the identified association between emotional state and honest behavior, we conducted a log-linear analysis. In accord with the results of the Fisher-Freeman-Halton test, a model assuming independence between emotion and cheating did not fit the data well, $G^{2}(2, \mathrm{~N}=156)=9.71, p=$ .008 , Cohen's $w=.22$. Of course, modeling an association between the two factors produced a saturated model that fit the data perfectly. However, if we treated the identified cell (i.e., grateful cheaters) as a structural void and modeled quasi-independence between emotion and cheating in the remaining cells, the model fit exceedingly well, $G^{2}(1, \mathrm{~N}=155)=0.01, p=.928$. This fact confirms that the association between emotional state and cheating was driven by the fewer than expected number of cheaters in the gratitude condition. Absent that cell, no support for an association between emotional state and cheating exists. Indeed, a Fisher's exact test comparing proportions of cheaters in the neutral and happiness conditions finds no reliable differences $(p=$ $1.00)$. 
We also intended to see whether gratitude would decrease cheating in a dose-dependent manner. That is, we wanted to determine whether differing intensities of gratitude would predict probabilities of behaving fairly, as this would provide additional support for our perspective. A logistic regression in which we regressed participants' choices to cheat or be fair on their levels of gratitude supported this view. In this model, gratitude proved to be a reliable predictor of behavior, $b=0.55,95 \% \mathrm{CI}: 0.13-0.96$, Wald $\chi^{2}=6.72, p=.010$, odds ratio $=1.73,95 \% \mathrm{CI}$ : $1.14-2.61$ (see Supplementary Materials for additional analyses). As can be seen in Figure 1, although the probability of behaving fairly hovered around $50 \%$ at low levels of gratitude, it increased to $95 \%$ at high levels (see Supplementary Materials for additional analyses).

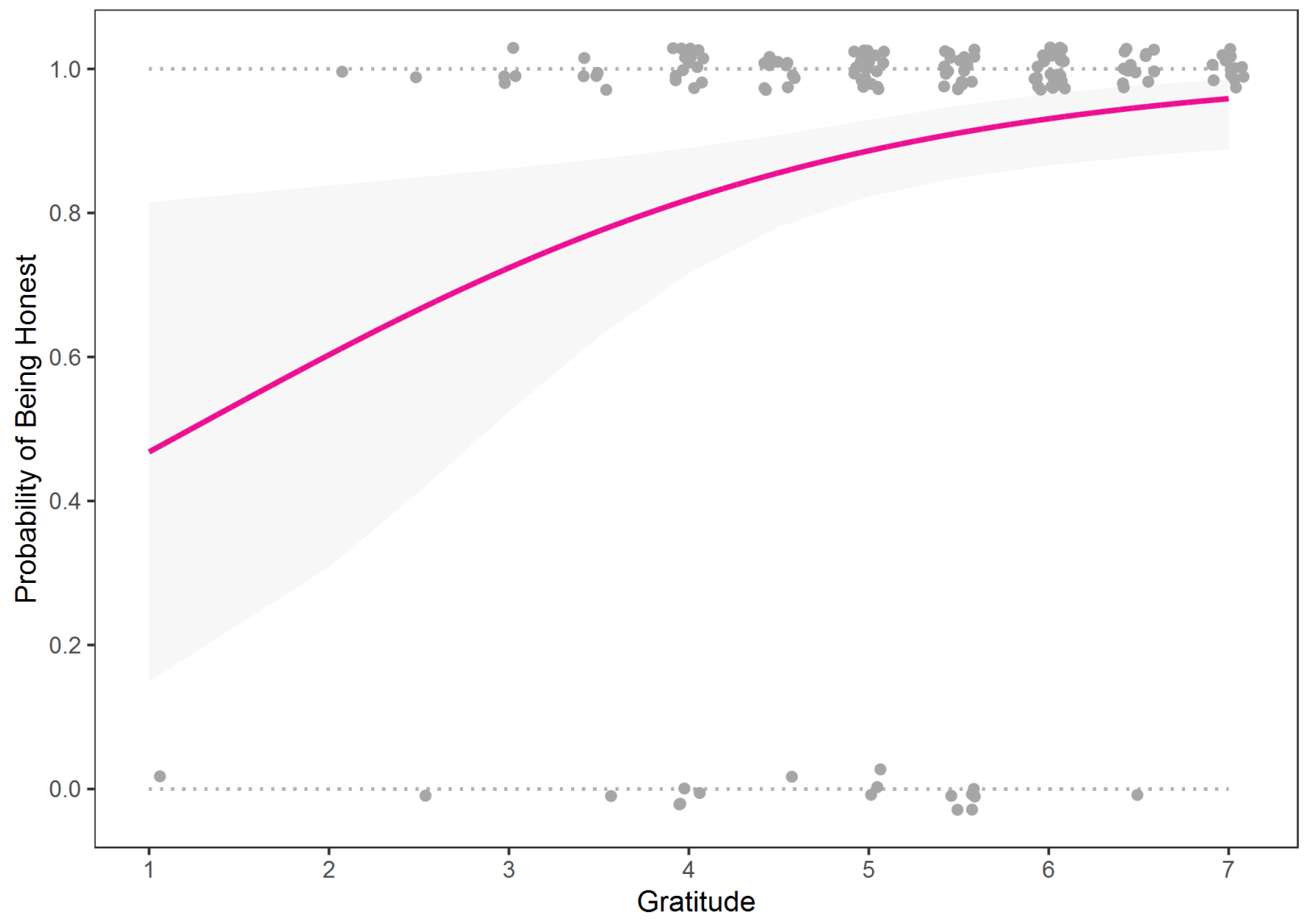

Figure 1. Probability of Behaving Fairly as a Function of Gratitude Intensity in Experiment 1. Note: Binary Choices Are Coded as Cheating $=0$, Behaving Honestly $=1$. Shaded area indicates 95\% CI. Points are Jittered. 
Finally, given that incremental gradations in gratitude directly predicted cheating behavior, we examined the indirect effect linking the gratitude manipulation to cheating via variance in such gradations. Identifying felt gratitude as a mediator in this manner would add significant support to our view, as it would indicate that the manipulation's effect on gratitude, as opposed to solely other aspects of the real-time encounter (e.g., a person - the benefactor in the gratitude condition - behaving prosocially toward the participant, a setting of a norm, etc.), directly influenced cheating behavior. We, therefore, used Hayes' PROCESS to examine the mediational model depicted in Figure 2. Here, emotion condition is dummy coded ( $1=$ gratitude, $0=$ others $)$ in a path model that allowed for the final endogenous variable (i.e., fair behavior) to be binary. PROCESS confirmed that both the direct $(2.10,95 \% \mathrm{CI}: 0.03-4.16)$ and indirect $(0.28,95 \% \mathrm{CI}$ : $0.01-0.70)$ effects of the emotion manipulation on behavior were significant, thereby confirming the causal efficacy of felt gratitude.

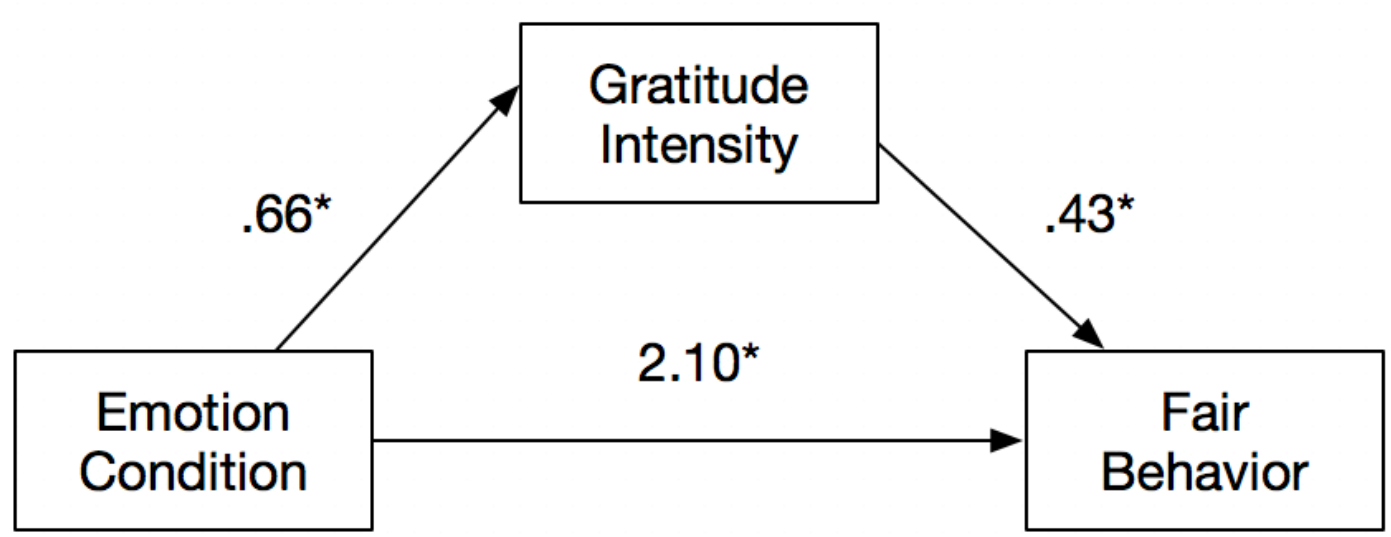

Figure 2. Path Model for Mediation by Gratitude (Emotion Condition dummy coded as Gratitude $=1$, Other $=0$; Fair Behavior dummy coded as Cheating $=0$, Fair Behavior $=1$ ) in Experiment 1 . Coefficients are raw, $* p<.05$. 


\section{Discussion}

This experiment lends support to the hypothesis that the gratitude people feel directly impacts their honesty. While only $2 \%$ of grateful participants cheated in assigning tasks to themselves, fully $16 \%$ of happy or neutral participants cheated. Moreover, gratitude appears to influence the probability of cheating in a dose-dependent way. Whereas participants experiencing high levels of gratitude could be expected to cheat only $5 \%$ of the time, those experiencing low levels could be expected to cheat almost $50 \%$ of the time. Of import, this experiment confirmed that this influence of gratitude was distinct; happiness, another positive affective state, did not produce a similar effect. Likewise, the mediation analysis showed that while certain aspects of the manipulation (i.e., receiving help from someone) affected cheating outside of the influence of gratitude, much of the manipulation's impact was directly mediated by alterations in this emotion. That is, holding constant the receipt of a favor, alterations in gratitude across the conditions predicted the probability of behaving fairly.

Although the findings were clear and in accord with the pre-registered predictions, we felt that three potential limitations remained. The first stemmed from the fact that if participants cheated in this experiment, they would not only be violating a moral rule but also harming another person, as they believed the next person to complete the experiment would be unfairly assigned to do a more onerous task. While this fact makes the choice to cheat or be fair more consequential, it also may confound the nature of gratitude's effect. One might argue that gratitude reduced cheating not because cheating violated a moral dictum, but solely because acting fairly prevented direct harm to another. 
The second limitation derived from the nature of the induction itself. Although our realtime method to induce gratitude possessed the benefit of being socially immersive, it also constrained the elicitor to be an act of someone offering immediate assistance. In daily life, gratitude, while necessitating a sense of obtaining something of value due to another's efforts, can be evoked in numerous ways that are not so temporally tied to an opportunity for immediate exchange (e.g., sharing time with a friend, the appreciation of natural beauty, the overcoming of illness).

The third limitation, which likely stemmed from the nature of the design, centered on the relatively low levels of cheating. While not a problem for our theory, the limited number of individuals who cheated decreased our ability to estimate cheating probability parameters with high accuracy.

To address these limitations, and provide a high-powered replication of the initial findings, we conducted a second experiment, that while closely mirroring the procedural dynamics of the first, utilized different methods of emotion induction and cheating.

\section{EXPERIMENT 2}

In this experiment, we recruited participants for what they believed was a study examining differences in recall for specific types of memories. In truth, descriptions of memories served as autobiographical recall emotion inductions. We also informed participants that everyone would be eligible to participate in a game of chance where they could earn additional money. At the end of the experiment, they would be able to flip a coin to determine how much extra remuneration they would receive and then report the result to the experimenter. Unbeknownst to them, the coin was fixed to initially indicate tails, meaning that they should receive the lower amount. Therefore, cheating here meant misreporting the results of the flip to achieve a preferred 
outcome, but unlike in Experiment 1, doing so did not directly harm another participant. As such, this measure of cheating was similar in design to others that have been frequently employed (cf. Lu et al., 2017; Lu, Lee, Gino, \& Galinsky, 2018).

\section{Methods}

\section{Open Practices Statement}

The experiment presented here is a conceptual replication of the preceding Experiment 1. It was not officially pre-registered, but adheres closely to the design and analytic plan of Experiment 1, which, as noted, was preregistered. Data files for this experiment will be posted with the pre-registered project at the URL noted for Experiment 1.

\section{Participants}

Given that this experiment is a conceptual replication of Experiment 1, which itself was highly powered with an obtained effect size similar to that anticipated in its preregistration, we aimed to recruit a similar sample size of 156 here for an a priori power $=.93$. Since participants would be recruited via Amazon Turk, we expected a loss of some due to the screening criteria we set (see below), and therefore set an initial recruitment goal of 180 .

Of the 180 participants, data from 39 (22\%) were removed from analysis because (a) they were from outside the United States (although we had noted only United States residents could take part, several individuals from other countries enrolled in the experiment), (b) they had nonresidential IP addresses (one recommended way to avoid data from bots on MTurk is to remove data coming from commercial IP addresses), (c) they failed to follow instructions (e.g., describing events other than the type requested in the autobiographical recall task), or (d) indicated strong suspicion. The final sample consisted of 141 participants (gender: 72 women, 67 men; Mean Age $=38$ years, $\mathrm{SD}=11.80)$. Prospective power for this sample size using a Cohen's 
$w=.30$ (noted in the pre-registration for Experiment 1 and justified above) is estimated to equal .90.

\section{Procedure}

As noted, participants believed that they were taking part in an experiment meant to examine differences for specific types of memories. They were informed that a type of memory would be randomly selected for them, and that once they were notified of its category, they were to recall one of the given types and to describe it in writing using as much detail as they could. Participants were given 5 minutes for this task, which served as an emotion induction via autobiographical recall (DeSteno et al., 2014).

When the allotted time had passed, participants completed an emotion manipulation check that was presented as a way to gauge their feelings after they recalled their respective specific types of memories. Participants then completed the honesty task, which was presented as a game of chance (see below), a series of demographic questions followed by a debriefing that also included a question in which participants were asked what they believed the experiment was about.

\section{Manipulations and Measures}

Emotion inductions. Based on random assignment, participants were told that they had been assigned to describe a time that they were grateful, happy, or to describe their typical day. As one might expect, the nature of the memories within each condition varied greatly. They were also told that they would have 5 minutes to write once they hit a key that indicated they had recalled the specified memory. At the end of 5 minutes of writing, they were automatically advanced to the emotion manipulation check. 
Emotion measures. Participants were next informed that we wanted to get a sense of how they felt at this moment (i.e., after recalling the memory). Therefore, they were to indicate the degree to which a series of emotion terms described their current feeling state using a 5-point scale $(1=$ not at all; $5=$ very much $)$. Gratitude was assessed as the mean response to two items: grateful and appreciative. Happiness was measured using the response to a single item: happiness. Note that due to a programming error, responses to the feeling indicator amused were not obtained in this experiment.

Cheating. Participants were informed that it was now time for the game of chance to determine extra compensation (note that recruitment information for this experiment had noted that a game of chance of some type to gain extra compensation would take place). Participants were told that everyone would receive some amount of extra money. However, to determine how much each person would receive in a fair manner, they would be required to flip a coin using an external web-based coin flipping site. A link was provided within the MTurk experiment for this third-party link. When participants clicked on this link, it opened a new window in their browser with an animated coin. When they clicked on the coin, it would spin before stopping to show heads or tails. Participants were told that if the coin ended on heads, they should report that and would receive an additional 40 cents. If it ended on tails, they should report that and would receive an additional 10 cents. They were also told that if they did not report their results, they wouldn't receive any extra compensation. Therefore, all participants returned to the MTurk window and indicated the supposed results of their coin flip.

In reality, the coin-flipping website (www.acoinflipper.com) was a site created by us in which the coin was rigged such that the first flip always ended on tails, after which it was randomized. We utilized a supposed third-party site to increase participants' confidence that the 
flip was anonymous (i.e., that we would not truly know what they had flipped). Because, similar to Experiment 1, everyone had been assigned to the less preferable condition, which here meant less additional compensation (10 cents), responses indicating that participants had flipped heads and were therefore to received 40 cents were coded as cheating.

\section{Results}

\section{Manipulation Check}

We submitted participants responses on the gratitude and happiness intensity scales to a 3 (Emotion Condition: Gratitude, Happiness, Neutral) X 2 (Emotion Intensity: Gratitude vs. Happiness) mixed ANOVA. As in Experiment 1, the emotion manipulations produced distinct states as indicated by a significant interaction, $F(2,138)=11.34, p<.001$. A one-way ANOVA on gratitude intensity proved significant, $F(2,138)=15.25, p<.001$, with Fisher's LSD comparisons $(\alpha=.05)$ confirming that those who completed the gratitude manipulation experienced significantly greater intensities of gratitude $(M=4.44,95 \% \mathrm{CI}: 4.21-4.66)$ than did those who completed the neutral $(M=3.37,95 \% \mathrm{CI}: 3.00-3.74)$ and happiness manipulations $(M=3.52,95 \%$ CI: $3.17-3.88)$.

Turning to happiness intensity, a similar one-way analysis confirmed that those who completed the happiness $(M=3.98,95 \% \mathrm{CI}: 3.64-4.32)$ and gratitude $(M=3.91,95 \% \mathrm{CI}: 3.63$ -4.19) manipulations reported greater intensities of happiness than did those who completed the neutral manipulation $(M=3.37,95 \% \mathrm{CI}: 3.02-3.72), F(2,138)=4.09, p=.019$. The fact that those in the gratitude and happiness conditions again reported similar levels of happiness but distinct levels of gratitude is to be expected, as the experience of gratitude itself usually cooccurs with positive feelings regarding a benefit received (Bartlett \& DeSteno, 2006; DeSteno et al., 2014). 


\section{Cheating}

Frequencies for cheating and behaving fairly as a function of emotional state are shown in Table 2. As expected, changes to the experimental design increased the overall cheating level compared to that of Experiment 1 (40\% vs. 12\%). However, also as in Experiment 1, a FisherFreeman-Halton exact test confirmed that an association between emotion condition and cheating was highly probable, with deviations in the proportion of cheating across emotion conditions as large or larger than that observed here only occurring by chance about two percent of the time $(p=.020)$. Examination of the adjusted standardized residuals clearly indicates that the principal deviations occurred among those feeling grateful, with the corresponding Pearson residuals pointing to the low number of cheaters in the gratitude condition as the larger discrepancy.

\begin{tabular}{lcccccccc}
\cline { 2 - 5 } Emotion & $\begin{array}{l}\text { Observed } \\
\text { Frequency }\end{array}$ & $\begin{array}{l}\text { Expected } \\
\text { Frequency }\end{array}$ & $\begin{array}{l}\text { Pearson } \\
\text { Residual }\end{array}$ & $\begin{array}{l}\text { Adj. Std. } \\
\text { Residual }\end{array}$ & $\begin{array}{l}\text { Observed } \\
\text { Frequency }\end{array}$ & $\begin{array}{l}\text { Expected } \\
\text { Frequency }\end{array}$ & $\begin{array}{l}\text { Pearson } \\
\text { Residual }\end{array}$ & $\begin{array}{l}\text { Adj. Std. } \\
\text { Residual }\end{array}$ \\
\hline \multirow{2}{*}{ Gratitude } & 15 & 22.6 & -1.6 & -2.7 & 41 & 33.4 & 1.3 & 2.7 \\
Neutral & 23 & 17.4 & 1.3 & 2.1 & 20 & 25.6 & -1.1 & -2.1 \\
Happiness & 19 & 17.0 & 0.5 & 0.8 & 23 & 25.0 & -0.4 & -0.8 \\
\hline
\end{tabular}

Table 2. Cheating as a Function of Emotion Condition in Experiment 2.

We again conducted a log-linear analysis to determine whether infrequent cheating in the gratitude condition explained the identified association between emotional state and honest 
behavior. In accord with the results of the Fisher-Freeman-Halton test, a model assuming independence between emotion and cheating fit the data poorly, $G^{2}(2, \mathrm{~N}=141)=7.94, p=.019$, Cohen's $w=.24$. As in Experiment 1, if we again treat the identified cell (i.e., grateful cheaters) as a structural void and model quasi-independence between emotion and cheating in the remaining cells, the model fits very well, $G^{2}(1, \mathrm{~N}=140)=0.58, p=.447$. No support for an association between emotional state and cheating exists when this cell is removed from the analysis. A Fisher's exact test comparing proportions of cheaters in the neutral and happiness conditions shows no reliable differences $(p=0.52)$.

We again wanted to determine if gratitude would decrease cheating in a dose-dependent way. Accordingly, we conducted a logistic regression in which we regressed participants' choices to cheat or be fair on their levels of gratitude. In this model, gratitude was again found to be a reliable predictor of behavior, $b=0.54,95 \% \mathrm{CI}: 0.23-0.86$, Wald $\chi^{2}=11.31, p=.001$, odds ratio $=1.72,95 \% \mathrm{CI}: 1.26-2.37$. As can be seen in Figure 3, although the probability of behaving fairly hovered around $25 \%$ at the lowest level of gratitude, it increased to approximate $75 \%$ at the highest level. 


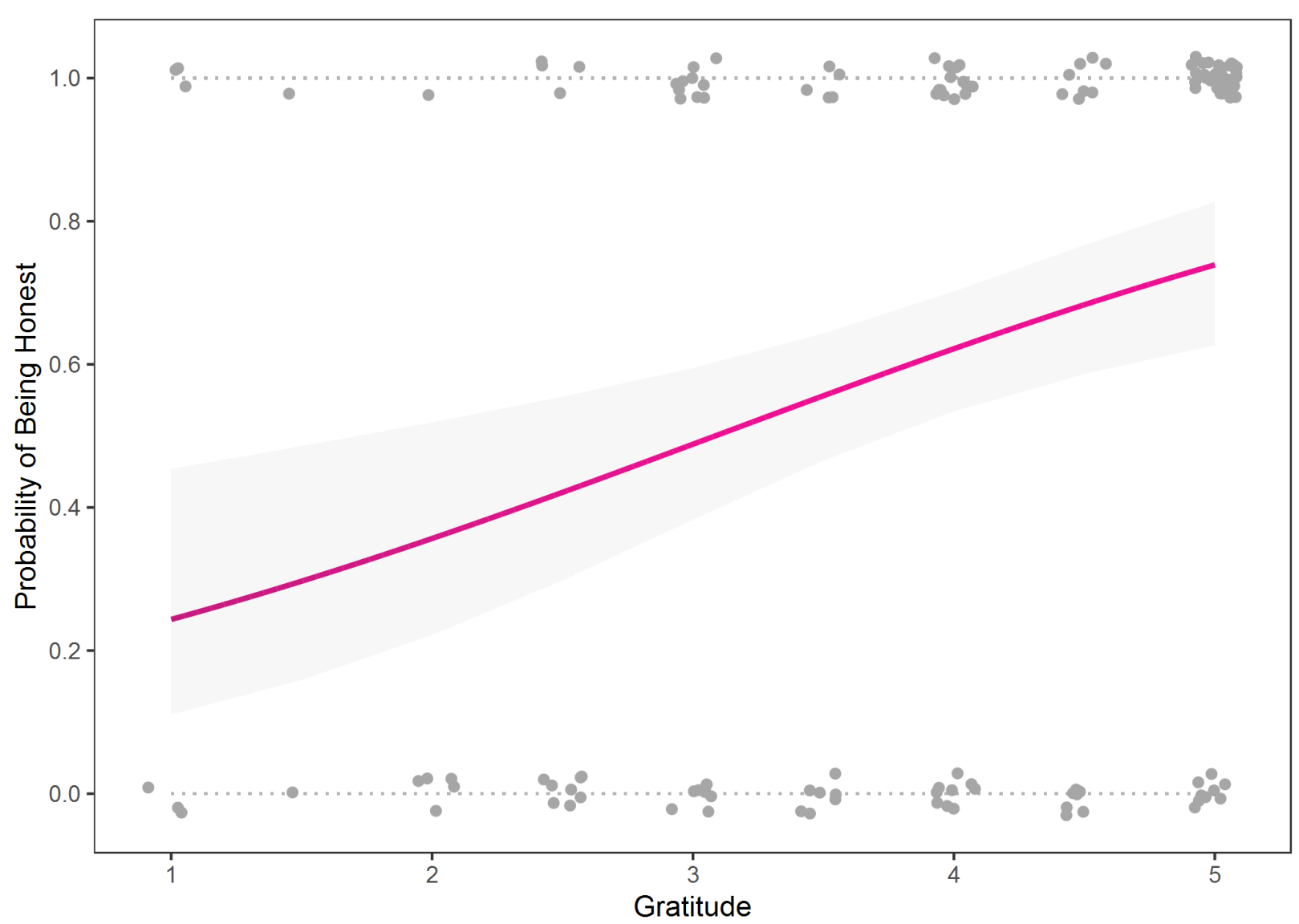

Figure 3. Probability of Behaving Fairly as a Function of Gratitude Intensity. Note: Binary Choices Are Coded as Cheating $=0$, Behaving Honestly $=1$. Shaded area indicates 95\% CI. Points are Jittered.

Finally, we again conducted a mediational analysis (see Figure 4) to examine the precise role played by gratitude in the causal chain in a path model that allowed for the final endogenous variable (i.e., fair behavior) to be binary. PROCESS confirmed that although the indirect effect of the emotion manipulation on behavior was significant $(0.44,95 \% \mathrm{CI}: 0.12-0.91)$, thereby confirming the causal efficacy of felt gratitude, the direct effect was not $(0.57,95 \% \mathrm{CI}:-0.23-$ 1.37). 


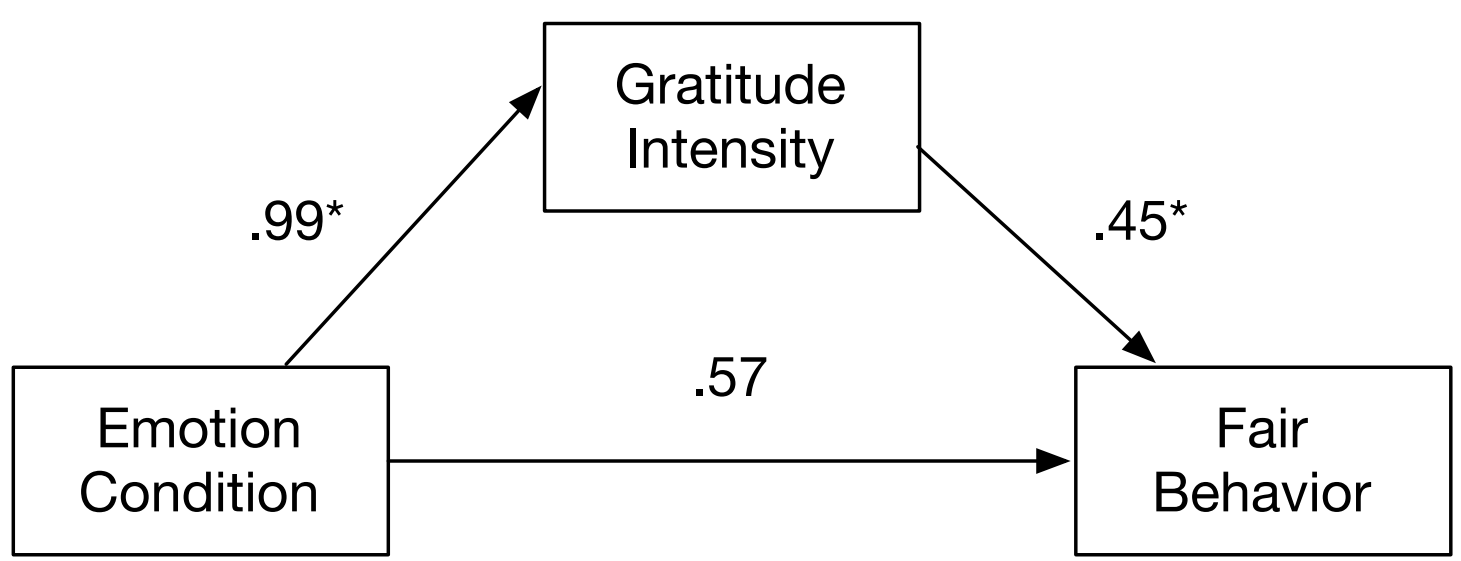

Figure 4. Path Model for Mediation by Gratitude (Emotion Condition dummy coded as Gratitude $=1$, Other $=0$; Fair Behavior dummy coded as Cheating $=0$, Fair Behavior $=1$ ) in Experiment 2. Coefficients are raw, ${ }^{*} p<.05$.

\section{Discussion}

As in Experiment 1, gratitude was found to enhance honest behavior thereby providing a close conceptual replication of the initial results. Whereas only $27 \%$ of those feeling grateful cheated to gain additional money, $53 \%$ and $45 \%$ of those feeling neutral or happy, respectively, did so. In addition, gratitude again predicted the probability of cheating in a dose-dependent way, here completely mediating the relation between emotional memories recalled and subsequent ethical behavior. Also as expected, the methodological alterations increased the overall level of cheating, thereby allowing enhanced accuracy in assessing this relation. While low levels of gratitude were associated with a probability of cheating around $75 \%$, high levels reduced that the probability to approximately $25 \%$.

\section{GENERAL DISCUSSION}

Taken together, the findings of Experiments 1 and 2 lend strong support to the hypothesis that gratitude enhances honest behavior. Not only did gratitude reduce cheating as compared to a neutral or a more general positively-valenced state like happiness, but did so in a dose-dependent way. This latter point is of great import, as it clearly identifies gratitude's causal role beyond that 
of any other elements involved in the induction process - a point supported by the very similar logistic regression effect sizes obtained across the differing paradigms used in Experiments 1 and 2 (odds ratios $=1.72$ and 1.73 , respectively). Moreover, as shown by the mediational analyses, even controlling for whether a person was offered help or recalled a time they received some benefit, variations in resulting gratitude directly influenced ethical behavior.

Although previous research has linked gratitude to economic cooperation, which itself entails some degree of trustworthiness, this work has been explicitly limited to interactions in which outcomes were dependent on joint decisions. For example, gratitude has been shown to increase people's willingness to cooperate in an economic game similar to the prisoner's dilemma (DeSteno et al., 2014). Such games, however, do not distinctly separate a motive to be honest from ones centered on the accumulation of joint gain or the reduction of harm. Here, especially in Experiment 2, we find evidence that gratitude directly alters the probability of cheating in a manner that solely involves an opportunity for selfish gain through dishonest means. That is, gratitude enhances honesty outside the realm of cooperation (i.e., where a person's outcomes depend on the fairness of another).

Given this relation between gratitude and cheating, gratitude stands as a promising candidate for an honesty "nudge" of sorts. Whereas many tactics used in schools or offices focus on instilling a motive to be honest through top-down mechanisms, gratitude appears capable of working form the bottom up. Enhancing honesty by reminding people that they should be honest, or that there are consequences to be paid for dishonesty, are effortful and inhibitory in nature. That is, they require people not only to remember to adhere to a moral code, but also to squelch any desire to behave unethically. Most emotion-based effects, however, work from the bottom up. That is, they change the automatic valuations and predictions people apply to stimuli in their 
environs and, correspondingly, how people interact with those stimuli (i.e., objects or options that offer costs or rewards (Barrett, 2017; Barrett \& Bar, 2009). Thus, gratitude may work rather effortlessly to alter people's decisions about the value of cheating, and as such, stem unethical behavior with less internal conflict. As a consequence, instilling a culture or habit of cultivating gratitude within a given environment could likely be expected to reduce cheating over time, much as its chronic experience has been shown to predict increased resistance to temptations for immediate gain (Dickens \& DeSteno, 2016).

This demonstration of gratitude's influence on a moral decision unrelated to exchange or joint outcomes also speaks to the important role this emotion might play in shaping many other types of moral action. Specifically, it opens the door to the possibility that virtues might clump in "good people" precisely because certain virtues give rise to others. We suspect this will be most likely for virtues, like gratitude, that enhance the ability to delay gratification. Although future work is needed to uncover the boundaries of gratitude's influence, the current findings offer initial support to the notion that gratitude can function as a just such a parent virtue, and one who's regular experience might offer a way to improve character not from the top down via willful adherence to specific moral dicta, but from the bottom up via emotion-induced motivations. 


\section{References}

Algoe, S. B. (2012). Find, remind, and bind: The functions of gratitude in everyday relationships. Social and Personality Psychology Compass, 6, 455-469.

Barrett, L. F. (2017). How Emotions are Made. Boston, MA: Houghton Mifflin Harcourt.

Barrett, L. F., \& Bar, M. (2009). See it with feeling: Affective predictions in the human brain. Royal Society Phil Trans B, 364, 1325-1334.

Bartlett, M. Y., \& DeSteno, D. (2006). Gratitude and prosocial behavior: Helping when it costs you. Psychological Science, 17, 319-325.

Batson, C. D., Kobrynowicz, D., Dinnerstein, J. L., Kampf, H. C., \& Wilson, A. D. (1997). In a very different voice: Unmasking moral hypocrisy. Journal of Personality and Social Psychology, 72, 1335.

Cicero, M. T. (2018). Pro Plancio. (H. W. Auden, Trans.). London: Forgotten Books. (Original work published $54 \mathrm{BCE})$.

DeSteno, D., Bartlett, M. Y., Baumann, J., Williams, L. A., \& Dickens, L. (2010). Gratitude as moral sentiment: Emotion-guided cooperation in economic exchange. Emotion, 10, 289293.

DeSteno, D., Condon, P., \& Dickens, L. (2016). Gratitude and compassion. In L.F. Barrett, M. Lewis, \& J.M. Haviland-Jones, Handbook of emotions $\left(5^{\text {th }}\right.$ ed.) (pp. 835-846). New York, NY: The Guilford Press.

DeSteno, D., Li, Y., Dickens, L., \& Lerner, J. S. (2014). Gratitude: A tool for reducing economic impatience. Psychological Science, 25, 1262-1267. 
Dickens, L., \& DeSteno, D. (2016). The grateful are patient: Heightened daily gratitude is associated with attenuated temporal discounting. Emotion, 16, 421-425.

Emmons, R.A. (2009). Gratitude. In D. Sander \& K. R. Scherer, Eds., Oxford Companion to the Affective Sciences (p. 198). New York, NY: Oxford University Press.

Frank, R. H. (1988). Passions within reason: The strategic role of the emotions. New York, NY: W.W. Norton \& Co.

King, G., \& Zeng, L. (2001). Logistic regression in rare events data. Political Analysis, 9, 137163.

Lu, J., Galinsky, A., Maddux, W., Chakroff, A., Quoidbach, J., \& Gino, F. (2017). The dark side of going abroad: How broad foreign experiences increase immoral behavior. Journal of Personality and Social Psychology, 112, 1-16.

Lu, J. G., Lee, J. J., Gino, F., \& Galinsky, A. D. (2018). Polluted morality: Air pollution predicts criminal activity and unethical behavior. Psychological Science, 29, 340-355.

Peterson, C., \& Seligman, M. E. (2004). Character strengths and virtues: A handbook and classification. Washington, DC: Oxford University Press.

Price, T. F., \& Harmon-Jones, E. (2016). Embodying approach motivation: A review of recent evidence. In A. J. Elliot (Ed.), Advances in Motivation Science, Volume 3 (pp. 81-111). Cambridge, MA: Academic Press.

Sinnott-Armstrong, W., \& Miller, C. (2017). Moral Psychology, Volume V: Virtue and Character. Cambridge, MA; London, UK: MIT Press.

Tsang, J. (2006). Gratitude and prosocial behavior: An experimental test of gratitude. Cognition and Emotion, 20, 138-148.

Valdesolo, P., \& DeSteno, D. (2007). Moral hypocrisy. Psychological Science, 18, 689-690. 
Valdesolo, P., \& DeSteno, D. (2008). The duality of virtue: Deconstructing the moral hypocrite. Journal of Experimental Social Psychology, 44, 1334-1338. 


\section{Author Contributions}

D. DeSteno developed the study concept. All authors contributed to the study design. Testing and data collection were performed by F. Duong, D. Lim, and S. Kates. Data analysis was conducted by D. DeSteno. D. DeSteno drafted the manuscript with F. Duong, D. Lim, and S. Kates providing critical revisions. All authors approved the final version of the manuscript for submission. 
Gratitude and Cheating 32

\section{Acknowledgements}

This research was funded by a grant from the John Templeton Foundation. 


\section{SOM-R}

Logistic regression analyses on data where one of the two binary responses is rare can produce biased estimates (King \& Zheng, 2001). Although the results of the analysis presented in the main text in Experiment 1 does not appear to show any hallmark signs of inflated standard errors or other estimation problems known to result from sparse data, we nonetheless decided to re-estimate the model here using an exact logistic regression given that cheating responses constituted only $12 \%$ of the binary behavioral responses. Exact logistic regression, although computationally intensive, is immune to the estimation problems that can stem from the analysis of sparse events. As expected, the results of this analysis (i.e., regressing cheating behavior on gratitude intensity) closely aligned with those of the logistic regression reported in the main text: $b=.541, p=.008$, odds ratio $=1.72,95 \% \mathrm{CI}: 1.13-2.65$.

Visual inspection of Figure 1 in main text might also raise concern that the point in the bottom left corner was overly influential. That is, given its position relative to the other cases, one might wonder whether it is a two-dimensional outlier that biased the steepness of the curve. To ensure that this was not the case, we calculated the studentized residual associated with this point and found it to be far from significant $(-1.23)$, thereby indicating that the point in question

did not overly influence the parameter estimates. Accordingly, the relation between gratitude and fair behavior remained significant in a re-analysis of the data with this one point removed. 